\section{GW23-e2689 ANALYSIS OF SAFETY OF ATORVASTATIN IN ASIAN PATIENTS IN CLINICAL TRIALS}

doi:10.1136/heartjnl-2012-302920ag.2

${ }^{1}$ Juliana Chan, ${ }^{2}$ Weihang Bao, ${ }^{2}$ Rana Fayyad, ${ }^{2}$ Rachel Laskey, ${ }^{1}$ Juliana Chan. ${ }^{1}$ Chinese University of Hong Kong, Hong Kong; ${ }^{2}$ Pfizer Inc, New York, USA

Objectives The benefits of atorvastatin therapy with respect to cardiovascular risk reduction have been well established through landmark clinical trials. These trials have also documented the safety of atorvastatin and have demonstrated that adverse events (AEs) are generally mild and transient.

Compared with evidence in Caucasian populations, the availability of statin safety data among Asian patients is limited. The objective of this retrospective analysis was to assess the safety of atorvastatin across the 10 to $80 \mathrm{mg}$ dose range among Asian patients from randomised clinical trials.

Methods Safety findings from 67637 patients in 55 atorvastatin clinical trials across the dose range $(10$ to $80 \mathrm{mg})$, including short term placebo-controlled (Median Exposure $(\mathrm{ME})=1$ year), activecomparator (ME<3 months), and dose-comparison ( $\mathrm{ME}<3$ months) studies were pooled, and analysed. Long-term cardiovascular outcome studies $(\mathrm{ME}=3.8$ to 4.9 years) were analysed separately.

Results The pooled database included a total of 3038 Asian patients (2445 atorvastatin; 217 placebo; 376 other) and 64599 non-Asian patients (42 348 atorvastatin, 7547 placebo, and 14704 other).

Overall, there was no evidence of increased rates of AEs in Asian patients when compared with the overall patient population. No cases of rhabdomyolysis were observed in atorvastatin-treated Asian patients. In patients receiving atorvastatin, the incidence of myalgia was 2.3\% (57/2445) in Asian patients versus 5.00\% (2235/ 44 793) overall.

Incidence of mild increases in LFTs $(1-1.2 \times$ ULN or up to $2 \times U L N)$ in the presence of a normal baseline reading, was similar between atorvastatin- and placebo-treated Asian patients.

Conclusions In this pooled analysis of atorvastatin clinical trial data, there was no evidence of increased incidence of all-causality AEs or myalgia in Asian patients compared with the overall population. 